\title{
DISKS EXTREMAL WITH RESPECT TO INTERPOLATION CONSTANTS
}

\author{
NGUyen Van Trao
}

\begin{abstract}
We define a function $\mu$ from the set of sequences in the unit ball to $\mathbb{R}_{+}^{*}$ by taking the greatest lower bound of the reciprocal of the interpolating constant of the sequences of the disk which get mapped to the given sequence by a holomorphic mapping from the disk to the ball. Its properties are studied in the spirit of the work of Amar and Thomas.
\end{abstract}

\section{Introduction}

Much attention has been given to the notion of interpolating sequences since L. Carleson introduced the concept in $[\mathbf{C}]$ and used it to establish a generalization of the Pick-Schwarz theorem (see e.g., $[\mathbf{C}],[\mathbf{V}],[\mathbf{B}]$, [B-C-L]).

In $[\mathbf{A}-\mathbf{T}]$ E. Amar and P. J. Thomas gave a new approach to the study of interpolating sequences in the unit ball $\mathbb{B}^{n}$ of $\mathbb{C}^{n}$, by considering maps from the unit disk to the ball, constrained to reach the points in the sequence, and extremal in the sense that the preimages in the disk should minimize the constant used by L. Carleson in his characterization of interpolating sequences in the disk $[\mathbf{C}]$. The aim of this paper is to continue this approach using the interpolation constant of the sequence of preimages. Although more technical to handle (there is no explicit formula, unlike in the work of Amar and Thomas), it should be more meaningful for the original problem of interpolation.

We now present more precisely the content of the paper. Let $a=$ $\left\{a_{k}\right\}_{k \in \mathbb{N}}$ a sequence of points in a domain $\Omega$ in $\mathbb{C}^{n}$, we say that $a$ is an interpolating sequence if, for any bounded sequence $v=\left\{v_{k}\right\}_{k \in \mathbb{N}}$, there is a bounded holomorphic function on $\Omega, f_{v}$, such that $f_{v}\left(a_{k}\right)=v_{k}$.

The constant of interpolation of $a$ is the smallest number $M_{a}^{\Omega}$ with $\forall v \in \ell^{\infty}(\mathbb{N}),\left\|f_{v}\right\|_{\infty} \leq M_{a}^{\Omega}\|v\|_{\infty}$. There always is a (finite) constant of interpolation when the sequence is interpolating. In particular, this makes sense for any finite sequence. 
E. Amar and P. J. Thomas defined for $a=\left\{a_{k}\right\}_{k \in \mathbb{N}} \subset \mathbb{B}^{n}$,

$$
\delta^{\mathbb{B}^{n}}(a):=\inf _{k} \prod_{j: j \neq k} d_{G}^{\mathbb{B}^{n}}\left(a_{k}, a_{j}\right),
$$

where $d_{G}^{\mathbb{B}^{n}}$ is the Gleason distance in $\mathbb{B}^{n}$ (in dimension 1, we denote $\delta^{\mathbb{D}}(a)$ instead of $\delta^{\mathbb{B}^{1}}(a)$ ), and

$$
\begin{aligned}
\rho(a):=\inf \left\{\delta^{\mathbb{D}}(\alpha): \alpha=\left\{\alpha_{k}\right\} \subset \mathbb{D},\right. & \exists \varphi \in \operatorname{Hol}\left(\mathbb{D}, \mathbb{B}^{n}\right) \\
& \text { such that } \left.\varphi\left(\alpha_{k}\right)=a_{k}, \forall k \in \mathbb{N}\right\},
\end{aligned}
$$

where $\mathbb{D}$ is the open unit ball in $\mathbb{C}$. We study the following analogue.

\section{Definition.}

$$
\begin{aligned}
\mu(a):=\inf \left\{\frac{1}{M_{\alpha}^{\mathbb{D}}}: \alpha\left\{\alpha_{j}\right\}_{j \in \mathbb{N}} \subset \mathbb{D}, \exists \varphi \in \operatorname{Hol}\left(\mathbb{D}, \mathbb{B}^{n}\right)\right. \\
\text { such that } \left.\varphi\left(\alpha_{k}\right)=a_{k}, \forall k \in \mathbb{N}\right\},
\end{aligned}
$$

where $M_{\alpha}^{\mathbb{D}}$ is the interpolation constant of $\alpha \subset \mathbb{D}$. Of course, this also applies to the special case of finite sequences, with $1 \leq j \leq N$.

It is easy to see that $\mu(a) \geq \frac{1}{M_{a}^{\mathbb{B}^{n}}}$. Maximizing $M_{\alpha}^{\mathbb{D}}$ among the sequences $\alpha \subset \mathbb{D}$ which are preimages of $a \subset \mathbb{B}^{n}$ yields mappings -if there are any - that are "the tightest" in the sense that it is more difficult to hit the points of $a$ with a map $\varphi$ from the disk to the ball when the preimage sequence $\alpha$ allows the interpolation by $H^{\infty}$ functions of fewer values, which is the intuitive meaning of a larger value of $M_{\alpha}^{\mathbb{D}}$.

We also know by Carleson $[\mathbf{C}]$, that for $\alpha \subset \mathbb{D}, M_{\alpha}^{\mathbb{D}} \geq \frac{1}{\delta^{\mathbb{D}}(\alpha)}$, thus $\mu(a) \leq \rho(a)$. We want to study the function $a \longmapsto \mu(a)$ in the same way that $\rho$ was studied in $[\mathbf{A}-\mathbf{T}]$ and $[\mathbf{T 1}]$. Namely, we will prove the following.

Theorem 2.7 (monotonicity). Let $a=\left\{a_{j}\right\}_{1 \leq j \leq N}$ be a sequence of points in $\mathbb{B}^{n}$ and $a^{\prime}$ be a subsequence of $a$. Then $\mu\left(a^{\prime}\right) \geq \mu(a)$.

Theorem 2.8 (continuity). $\mu(a)$ depends continuously on $a$ in the set of finite sequences.

Theorem 3.1 (approximation by finite sequences). Let $a=\left\{a_{k}\right.$, $\left.k \in Z_{+}^{*}\right\} \subset \mathbb{B}^{n}$. Then

$$
\mu(a)=\lim _{N \rightarrow \infty} \mu\left(\left\{a_{j}\right\}_{1 \leq j \leq N}\right)=\inf _{N \in Z_{+}^{*}} \mu\left(\left\{a_{j}\right\}_{1 \leq j \leq N}\right) .
$$


The next theorem shows that there is a large set of sequences for which our infimum is in fact a minimum.

Theorem 3.5. For any $N \in Z_{+}^{*}$ there exists an open set $U$ of sequences a with $\# a=N$ such that for every $a \in U$ there is $\alpha=\left\{\alpha_{j}\right\}_{1 \leq j \leq N} \subset \mathbb{D}$ such that $\varphi\left(\alpha_{j}\right)=a_{j}$ for $1 \leq j \leq N$ and $\mu(a)=\frac{1}{M_{\alpha}^{\mathbb{D}}}$.

Finally, we connect our definition with a more classical extremal problem, which shows that some form of uniqueness holds for the extremal mappings: when the $\alpha_{j}$ are given, the map $\varphi_{0}$ is unique.

Theorem 3.6. Let $a=\left\{a_{j}\right\}_{1 \leq j \leq N}$ be a sequence of points in $\mathbb{B}^{n}$. If there exists a holomorphic map $\varphi_{0}$ from $\mathbb{D}$ to $\mathbb{B}^{n}$ and $\alpha=\left\{\alpha_{j}\right\}_{1 \leq j \leq N} \subset \mathbb{D}$ such that $\varphi_{0}\left(\alpha_{j}\right)=a_{j}$ for $1 \leq j \leq N$ and $\mu(a)=\frac{1}{M_{\alpha}^{\mathbb{D}}}$, then $\varphi_{0}$ is a solution of the extremal problem

$$
\inf \left\{\|\psi\|_{\infty}: \psi \in H\left(\mathbb{D}, \mathbb{C}^{n}\right), \psi\left(\alpha_{j}\right)=a_{j} \text { for } 1 \leq j \leq N\right\}
$$

We remark that some of the methods used here can be applied (indeed, in a simpler way) to simplify the proofs of [T1].

\section{Definitions and notations}

$$
\begin{aligned}
\text { For } z, w \in \mathbb{C}^{n}, z \cdot \bar{w}:=\sum_{j=1}^{n} z_{j} \bar{w}_{j},|z|^{2}:=z \cdot \bar{z} \\
\mathbb{B}^{n}:=\left\{z \in \mathbb{C}^{n} \text { such that }|z|<1\right\}, \quad \mathbb{D}:=\mathbb{B}^{1} .
\end{aligned}
$$

Let $\Omega$ be a domain in $\mathbb{C}^{n}$ and $z, w \in \Omega$, the Gleason (or invariant) distance $d_{G}^{\Omega}(z, w)$ is given by $d_{G}^{\Omega}(z, w)=\sup \left\{|f(z)|: f \in H^{\infty}(\Omega)\right.$ such that $\left.f(w)=0,\|f\|_{\infty} \leq 1\right\}$. We know that

$$
1-d_{G}^{\mathbb{B}^{n}}(z, w)^{2}:=\frac{\left(1-|z|^{2}\right)\left(1-|w|^{2}\right)}{|1-z \cdot \bar{w}|^{2}}
$$

in particular,

$$
d_{G}^{\mathbb{D}}(z, w)=\frac{|z-w|}{|1-z \bar{w}|}
$$


For $\lambda \in \mathbb{D}$ let us denote by $\Phi_{\lambda}$ the Möbius map of $\mathbb{D}$ defined as follows:

$$
\Phi_{\lambda}(\zeta):=\frac{\lambda-\zeta}{1-\bar{\lambda} \zeta} .
$$

(See $[\mathbf{G} \mathbf{1}],[\mathbf{R}]$.

Finally, for $f$ a bounded holomorphic function from $\mathbb{D}$ to $\mathbb{C}^{n}$ we write

$$
f \in H_{n}^{\infty}(\mathbb{D}), \quad\|f\|_{\infty}^{2}:=\sup _{z \in \mathbb{D}}\left(\left|f_{1}(z)\right|^{2}+\cdots+\left|f_{n}(z)\right|^{2}\right) .
$$

\section{Continuity of the function $\mu$ in the finite case}

For the beginning we need some easy lemma.

Lemma 2.1. For any $\beta_{1}, \ldots, \beta_{p} \in \partial \mathbb{D}, \delta>0$, there exists a function $h_{\delta} \in A(\overline{\mathbb{D}})$ (i.e continuous up to the boundary) with $\left\|h_{\delta}\right\|_{\infty}=1$, such that

(1) $h_{\delta}\left(\beta_{j}\right)=1, \quad 1 \leq j \leq p$;

(2) $\left|h_{\delta}(\zeta)\right| \leq \delta, \quad \forall \zeta \in \overline{\mathbb{D}} \backslash \bigcup_{j=1}^{p} D\left(\beta_{j}, \delta\right)$.

Proof: Remark that every finite set in $\partial \mathbb{D}$ is a peak-interpolation set. Let $g \in A(\overline{\mathbb{D}})$ be such that $g\left(\beta_{j}\right)=1,1 \leq j \leq p$, and $|g(\zeta)|<1$, $\forall \zeta \in \overline{\mathbb{D}} \backslash\left\{\beta_{j}, 1 \leq j \leq p\right\}$. For $N$ large enough, $g^{N}$ satisfies the required properties.

Lemma 2.2. With the hypotheses of Lemma 2.1, there exists $f \in A(\overline{\mathbb{D}})$, $\|f\|_{\infty} \leq 1$ such that

(1) $f\left(\beta_{j}\right)=0, \quad 1 \leq j \leq p$;

(2) $|f(\zeta)-1| \leq \delta, \quad \forall \zeta \in \overline{\mathbb{D}} \backslash \bigcup_{j=1}^{p} D\left(\beta_{j}, \delta\right)$.

Proof: Take $\delta_{1} \in(0, \delta]$ and $g=h_{\delta_{1}}$ as in Lemma 2.1. Now, for $\delta_{2}>0$, let $f(\zeta)=\Phi_{1-\delta_{2}}\left(\left(1-\delta_{2}\right) g(\zeta)\right)=\frac{\left(1-\delta_{2}\right)(1-g(\zeta))}{1-\left(1-\delta_{2}\right)^{2} g(\zeta)}$, then $f\left(\beta_{j}\right)=0$ and $\|f\|_{\infty} \leq 1$ by construction. Since

$$
f(\zeta)-1=\frac{-\delta_{2}\left(1+\left(1-\delta_{2}\right) g(\zeta)\right)}{1-\left(1-\delta_{2}\right)^{2} g(\zeta)}
$$

for $|g(\zeta)| \leq \delta_{1},|f(\zeta)-1| \leq \frac{\delta_{2}\left(1+\delta_{1}\left(1-\delta_{2}\right)\right)}{1-\delta_{1}\left(1-\delta_{2}\right)^{2}} \leq \delta_{2} \frac{1+\delta_{1}}{1-\delta_{1}}<\delta$ for an appropriate choice of $\delta_{2}$. 
Lemma 2.3. Let $\alpha=\left\{\alpha_{j}, j \in J\right\}$ and $J=J_{1} \cup J_{2}$ with $J_{1} \cap J_{2}=\emptyset$, $J_{1} \neq \emptyset, J_{2} \neq \emptyset$. Set $\alpha^{(1)}=\left\{\alpha_{j}, j \in J_{1}\right\}, \alpha^{(2)}=\left\{\alpha_{j}, j \in J_{2}\right\}, M_{1}=$ $M_{\alpha^{(1)}}^{\mathbb{D}}, M_{2}=M_{\alpha^{(2)}}^{\mathbb{D}}$. Assume further that $\alpha^{(1)} \subset D(0, r) \subset \subset \mathbb{D}$, and there exist $\beta_{j} \in \partial \mathbb{D}$, for $j \in J_{2}$, such that $\left|\alpha_{j}-\beta_{j}\right| \leq \delta, j \in J_{2}$. Fix $M>0$. Then for any $g_{1}, g_{2} \in H^{\infty}\left(\mathbb{D}, \mathbb{C}^{n}\right)$ and $\left\|g_{1}\right\|_{\infty} \leq M,\left\|g_{2}\right\|_{\infty} \leq$ $M$, and $\varepsilon>0$, there exists $\delta_{0}$ such that for any $\delta<\delta_{0}$ and $\alpha$ as above, there exists $f \in H^{\infty}\left(\mathbb{D}, \mathbb{C}^{n}\right)$ and $f\left(\alpha_{j}\right)=g_{\ell}\left(\alpha_{j}\right), j \in J_{\ell}, \ell=1,2$, and $\|f\|_{\infty} \leq \max \left(\left\|g_{1}\right\|_{\infty},\left\|g_{2}\right\|_{\infty}\right)+\varepsilon$

Proof: By Lemma 2.2, there exists a function $h_{1}$ associated to $\delta_{1}$, which we choose smaller than $\frac{1-r}{2}$ and $\varepsilon_{1}$, where $\varepsilon_{1}>0$ is to be chosen later. Choose $\delta_{2}<\delta_{1}$ small enough so that $\delta_{2} \leq \varepsilon_{1}$ and $\min _{j}\left|\zeta-\beta_{j}\right| \leq \delta_{2}$ implies that $\left|h_{1}(\zeta)\right| \leq \varepsilon_{1}$. Let $h_{2}$ be the function obtained from Lemma 2.1 applied with $\delta=\delta_{2}$. We pick $\delta_{0}$ small enough so that $\min _{j}\left|\zeta-\beta_{j}\right| \leq \delta_{0}$ implies that $\left|h_{2}(\zeta)-1\right| \leq \varepsilon_{1}$.

Now consider the function $f_{1}(\zeta)=h_{1}(\zeta) g_{1}(\zeta)+h_{2}(\zeta) g_{2}(\zeta)$. For $\min _{j}\left|\zeta-\beta_{j}\right| \leq \delta_{2},\left|h_{1}(\zeta)\right| \leq \varepsilon_{1},\left|h_{2}(\zeta)\right| \leq 1$, so

$$
\left\|f_{1}(\zeta)\right\| \leq \varepsilon_{1}\left\|g_{1}\right\|_{\infty}+\left\|g_{2}\right\|_{\infty} \leq\left(1+\varepsilon_{1}\right) \max \left(\left\|g_{1}\right\|_{\infty},\left\|g_{2}\right\|_{\infty}\right) .
$$

For $\min _{j}\left|\zeta-\beta_{j}\right| \geq \delta_{2},\left|h_{2}(\zeta)\right| \leq \delta_{2} \leq \varepsilon_{1},\left|h_{1}(\zeta)\right| \leq 1$, so

$$
\left\|f_{1}(\zeta)\right\| \leq\left\|g_{1}\right\|_{\infty}+\varepsilon_{1}\left\|g_{2}\right\|_{\infty} \leq\left(1+\varepsilon_{1}\right) \max \left(\left\|g_{1}\right\|_{\infty},\left\|g_{2}\right\|_{\infty}\right) .
$$

On the other hand, for $j \in J_{1}$,

$$
\begin{aligned}
\left\|f_{1}\left(\alpha_{j}\right)-g_{1}\left(\alpha_{j}\right)\right\| & =\left\|\left(h_{1}\left(\alpha_{j}\right)-1\right) g_{1}\left(\alpha_{j}\right)+h_{2}\left(\alpha_{j}\right) g_{2}\left(\alpha_{j}\right)\right\| \\
\leq & \varepsilon_{1}\left\|g_{1}\right\|_{\infty}+\varepsilon_{1}\left\|g_{2}\right\|_{\infty} \leq 2 \varepsilon_{1} \max \left(\left\|g_{1}\right\|_{\infty},\left\|g_{2}\right\|_{\infty}\right) ;
\end{aligned}
$$

for $j \in J_{2}$,

$$
\begin{aligned}
\left\|f_{1}\left(\alpha_{j}\right)-g_{2}\left(\alpha_{j}\right)\right\|= & \left\|h_{1}\left(\alpha_{j}\right) g_{1}\left(\alpha_{j}\right)+\left(h_{2}\left(\alpha_{j}\right)-1\right) g_{2}\left(\alpha_{j}\right)\right\| \\
& \leq \varepsilon_{1}\left\|g_{1}\right\|_{\infty}+\varepsilon_{1}\left\|g_{2}\right\| \leq 2 \varepsilon_{1} \max \left(\left\|g_{1}\right\|_{\infty},\left\|g_{2}\right\|_{\infty}\right) .
\end{aligned}
$$

We can find a map $f_{2}$ such that $f_{2}\left(\alpha_{j}\right)=g_{\ell}\left(\alpha_{j}\right)-f_{1}\left(\alpha_{j}\right), \forall j \in J_{\ell}$, $\ell=1,2$, and $\left\|f_{2}\right\|_{\infty} \leq 2 \varepsilon_{1} \sqrt{n} M_{\alpha}^{\mathbb{D}} \max \left(\left\|g_{1}\right\|_{\infty},\left\|g_{2}\right\|_{\infty}\right)$ (where $M_{\alpha}^{\mathbb{D}}$ is the constant of interpolation of $\alpha$, and it is well known $M_{\alpha}^{\mathbb{D}}$ is bounded by $\widetilde{M}$, which is depending only on $M_{1}, M_{2}$, and $(1-r) / 2$, supposing as we may that $\left.\delta \leq \delta_{0} \leq(1-r) / 2\right)$. Setting $f=f_{1}+f_{2}$, we have $f\left(\alpha_{j}\right)=g_{\ell}\left(\alpha_{j}\right)$, 
$\forall j \in J_{\ell}, \ell=1,2$, and

$$
\begin{aligned}
& \begin{aligned}
\|f\|_{\infty} & \leq\left(1+\varepsilon_{1}\right) \max \left(\left\|g_{1}\right\|_{\infty},\left\|g_{2}\right\|_{\infty}\right)+2 \varepsilon_{1} \sqrt{n} \widetilde{M} \max \left(\left\|g_{1}\right\|_{\infty},\left\|g_{2}\right\|_{\infty}\right) \\
& =\max \left(\left\|g_{1}\right\|_{\infty},\left\|g_{2}\right\|_{\infty}\right)+\varepsilon_{1} \max \left(\left\|g_{1}\right\|_{\infty},\left\|g_{2}\right\|_{\infty}\right)(1+2 \sqrt{n} \widetilde{M}) \\
& \leq \max \left(\left\|g_{1}\right\|_{\infty},\left\|g_{2}\right\|_{\infty}\right)+\varepsilon_{1} M(1+2 \sqrt{n} \widetilde{M})
\end{aligned} \\
& \text { Taking } \varepsilon_{1}=\frac{\varepsilon}{M(1+2 \sqrt{n} \widetilde{M})}, \text { the property is proved. }
\end{aligned}
$$

The next result follows immediately from Lemma 2.3.

Corollary 2.4. Suppose $\alpha=\left\{\alpha_{j}, j \in J\right\}$ and $J=J_{1} \cup J_{2}$ with $J_{1} \cap J_{2}=$ $\emptyset, J_{1} \neq \emptyset, J_{2} \neq \emptyset$. Set $\alpha^{(1)}=\left\{\alpha_{j}, j \in J_{1}\right\}, \alpha^{(2)}=\left\{\alpha_{j}, j \in J_{2}\right\}$, $M_{1}=M_{\alpha^{(1)}}^{\mathbb{D}}, M_{2}=M_{\alpha^{(2)}}^{\mathbb{D}}$. Assume further that $\alpha^{(1)} \subset D(0, r) \subset \subset \mathbb{D}$, and there exist $\beta_{j} \in \partial \mathbb{D}$, for $j \in J_{2}$, such that $\left|\alpha_{j}-\beta_{j}\right| \leq \delta, j \in J_{2}$. Then for any $\varepsilon>0$, there exists $\delta_{0}$ such that for any $\delta<\delta_{0}$ and $\alpha$ as above, $\max \left(M_{1}, M_{2}\right) \leq M_{\alpha}^{\mathbb{D}} \leq \max \left(M_{1}, M_{2}\right)+\varepsilon$.

Proof: Take $n=1$; given values $\left\{v_{j}, j \in J\right\} \subset \overline{\mathbb{D}}$ to interpolate, we know there exist functions $g_{1}, g_{2} \in H^{\infty}(\mathbb{D}), g_{\ell}\left(\alpha_{j}\right)=v_{j}, j \in J_{\ell}$ and $\|g\|_{\infty} \leq M_{\ell}, \ell=1,2$. Applying Lemma 2.3 we have the property.

The following lemma is our main technical tool, inspired to some extent by the work of Globevnik [G2].

Lemma 2.5. Let $a=\left\{a_{j}\right\}_{1 \leq j \leq N}$ be a sequence of points in $\mathbb{B}^{n}$. Suppose that there exist a sequence $\alpha=\left\{\alpha_{j}\right\}_{1 \leq j \leq N}$ in $\mathbb{D}$ and a holomorphic map $\varphi: \mathbb{D} \rightarrow \mathbb{B}^{n}$ such that $a_{j}=\varphi\left(\alpha_{j}\right)$ for $1 \leq j \leq N$. Let $a_{N+1}$ be a point of $\mathbb{B}^{n}$ and $\varepsilon>0$. Then there exist $\alpha^{\prime}=\left\{\alpha_{j}^{\prime}\right\}_{1 \leq j \leq N} \subset \mathbb{D}$ and $\beta \in \mathbb{D}$ and holomorphic map $\psi$ from $\mathbb{D}$ into $\mathbb{B}^{n}$ such that $\psi\left(\alpha_{j}^{\prime}\right)=a_{j}, \psi(\beta)=a_{N+1}$ and $M_{\alpha^{\prime} \cup\{\beta\}}^{\mathbb{D}} \geq M_{\alpha}^{\mathbb{D}}-\varepsilon$.

Proof: For all $r<1$ such that $\alpha / r \subset \mathbb{D}, M_{\alpha / r}^{\mathbb{D}} \leq M_{\alpha}^{\mathbb{D}}$, and we know by the continuity of the interpolation constant that $M_{\alpha / r}^{\mathbb{D}}$ tends to $M_{\alpha}^{\mathbb{D}}$ as $r$ tends to 1 .

Let $\max \left(\left|\alpha_{1}\right|, \ldots,\left|\alpha_{N}\right|\right)<r<1$ and $s=\max \left(\left|a_{1}\right|, \ldots,\left|a_{N}\right|,\left|a_{N+1}\right|\right.$, $\sup |\varphi|)$. Apply Lemma 2.3 with $g_{1}(\zeta)=\varphi(r \zeta),\left\|g_{1}\right\|_{\infty}=\sup |\varphi| \leq$ $\overline{D_{r}}$ $\overline{D_{r}}$

$s<1 ; g_{2}(\zeta)=a_{N+1}, \forall \zeta \in \mathbb{D},\left\|g_{2}\right\|_{\infty}=\left|a_{N+1}\right| \leq s ; \alpha_{j}^{\prime}=\alpha_{j} / r \in \mathbb{D}$ for $j \in J_{1}:=\{1, \ldots, N\} ; J_{2}:=\{N+1\}$, and the additional one-point sequence is chosen as $\alpha_{N+1}^{\prime}:=1-\delta\left(\varepsilon_{1}\right)$, where $\varepsilon_{1}=\varepsilon_{1}(r):=1-s$, and $\delta\left(\varepsilon_{1}\right)<\delta_{0}$, where $\delta_{0}$ is given by Lemma 2.3 . 
Take $\Psi^{\left(\varepsilon_{1}\right)}=f$ in Lemma 2.3 ; then we have $\Psi^{\left(\varepsilon_{1}\right)}\left(\alpha_{j}^{\prime}\right)=g_{1}\left(\alpha_{j}^{\prime}\right)=a_{j}$, $1 \leq j \leq N$, and $\Psi^{\left(\varepsilon_{1}\right)}\left(\alpha_{N+1}^{\prime}\right)=g_{2}\left(\alpha_{N+1}^{\prime}\right)=a_{N+1} ;\left\|\Psi^{\left(\varepsilon_{1}\right)}\right\|_{\infty} \leq s+\varepsilon_{1}=$ 1 .

Now $M_{\alpha^{\prime}}^{\mathbb{D}} \geq M_{\alpha / r}^{\mathbb{D}}$, which can be made arbitrarily close to $M_{\alpha}^{\mathbb{D}}$ by the considerations at the beginning of the proof.

Our lemma is completely proved.

We now remark that the solution to our extremal problem is not modified if we only require the map $\varphi$ to hit only a subset of the sequence $\left\{a_{j}\right\}$. This would also hold for the original extremal problem in $[\mathbf{A}-\mathbf{T}]$. The quantity $\mu(a)$ is defined in the introduction.

Theorem 2.6. Let $a=\left\{a_{j}\right\}_{1 \leq j \leq N}$ be a sequence of points in $\mathbb{B}^{n}$. Then

$$
\begin{aligned}
& \mu(a)=\inf \left\{\frac{1}{M_{\alpha}^{\mathbb{D}}}: \exists J \subset\{1,2, \ldots, N\}, \exists \varphi \in H\left(\mathbb{D}, \mathbb{B}^{n}\right)\right. \\
& \left.\qquad \text { and } \alpha=\left\{\alpha_{j}\right\}_{j \in J} \subset \mathbb{D} \text { s.t. } \varphi\left(\alpha_{j}\right)=a_{j}, \forall j \in J\right\} .
\end{aligned}
$$

Proof: By using Lemma 2.5 repeatedly to add one point at a time to the set $\left\{a_{j}, j \in J\right\}$, we have

$$
\begin{aligned}
\mu(a)= & \inf \left\{\frac{1}{M_{\alpha}^{\mathbb{D}}}: \alpha \subset \mathbb{D}, \exists \varphi \in H\left(\mathbb{D}, \mathbb{B}^{n}\right) \text { s.t. } \varphi\left(\alpha_{j}\right)=a_{j}, \quad 1 \leq j \leq N\right\} \\
\leq & \inf \left\{\frac{1}{M_{\alpha}^{\mathbb{D}}}: \exists J \subset\{1,2, \ldots, N\}, \exists \varphi \in H\left(\mathbb{D}, \mathbb{B}^{n}\right)\right. \\
& \text { and } \left.\alpha=\left\{\alpha_{j}\right\}_{j \in J} \subset \mathbb{D} \text { such that } \varphi\left(\alpha_{j}\right)=a_{j}, \forall j \in J\right\} .
\end{aligned}
$$

The converse inequality is obvious and, hence Theorem 2.6 is proved.

Applying the above theorem we have the following.

Theorem 2.7. Let $a=\left\{a_{j}\right\}_{1 \leq i \leq N}$ be a sequence of points in $\mathbb{B}^{n}$ and $a^{\prime}$ be a subsequence of $a$. Then $\mu\left(a^{\prime}\right) \geq \mu(a)$.

Now we consider the continuity of the map $a \longmapsto \mu(a)$.

Theorem 2.8. $\mu(a)$ depends continuously on $a$ in the set of finite sequences.

Proof. First of all we prove the upper semi continuity of the map $a \longmapsto$ $\mu(a)$. 
Let $a=\left\{a_{j}\right\}_{1 \leq j \leq N} \subset \mathbb{B}^{n}$. We shall prove that $\forall \varepsilon>0, \exists \eta>$ 0 such that $\mu\left(a^{\prime}\right) \leq \mu(a)+\varepsilon$ for every $a^{\prime}=\left\{a_{j}^{\prime}\right\}_{1 \leq j \leq N} \subset \mathbb{B}^{n}$ with $\max _{1 \leq j \leq N}\left|a_{j}-a_{j}^{\prime}\right| \leq \eta$.

Let $\alpha=\left\{\alpha_{j}\right\}_{1 \leq j \leq N}$ be a sequence in $\mathbb{D}$ and $\varphi$ be a map from $\mathbb{D}$ to $\mathbb{B}^{n}$ such that $\varphi\left(\alpha_{j}\right)=a_{j}$ and $\frac{1}{M_{\alpha}^{\mathbb{D}}}<\mu(a)+\frac{\varepsilon}{2}$.

For $r<1$, put $\varphi_{r}(\zeta)=\varphi(r \zeta)$. Then

$$
\varphi_{r}\left(\frac{\alpha_{j}}{r}\right)=a_{j} \quad \text { and } \quad\left\|\varphi_{r}\right\|_{\infty}=\sup _{\bar{D}(0, r)}|\varphi|<1 .
$$

Since $M_{\alpha}^{\mathbb{D}}$ depends continuously on $\alpha$ (see [T2, Lemma 1]), we have $\frac{1}{M_{\alpha / r}^{\mathbb{D}}} \leq \frac{1}{M_{\alpha}^{\mathbb{D}}}+\frac{\varepsilon}{2}$ for $r$ large, where $\frac{\alpha}{r}=\left\{\frac{\alpha_{j}}{r}\right\}_{1 \leq j \leq N}$. Now there exists a map $f_{r}$ from $\mathbb{D}$ to $\mathbb{B}^{n}$ such that $f_{r}\left(\frac{\alpha_{j}}{r}\right)=a_{j}^{\prime}-a_{j}$ and $\left\|f_{r}\right\|_{\infty} \leq$ $\eta \sqrt{n} M_{\alpha / r}^{\mathbb{D}} \leq \eta \sqrt{n} M_{\alpha}^{\mathbb{D}}$.

Then $\left(f_{r}+\varphi_{r}\right)\left(\frac{\alpha_{j}}{r}\right)=a_{j}^{\prime}$ and $\left\|f_{r}+\varphi_{r}\right\|_{\infty} \leq\left\|\varphi_{r}\right\|_{\infty}+\eta \sqrt{n} M_{\alpha}^{\mathbb{D}}<1$ for $\eta$ small enough. It implies that

$$
\mu\left(a^{\prime}\right) \leq \frac{1}{M_{\alpha / r}^{\mathbb{D}}} \leq \frac{1}{M_{\alpha}^{\mathbb{D}}}+\frac{\varepsilon}{2}<\mu(a)+\varepsilon
$$

We now prove the lower semi continuity of the map $\mu(a)$.

Assume that $a^{k}=\left\{a_{j}^{k}\right\}_{j=1}^{N} \subset \mathbb{B}^{n}$ such that $a_{j}^{k} \rightarrow a_{j}$ as $k \rightarrow \infty$. We shall show that $\liminf _{k \rightarrow \infty} \mu\left(a^{k}\right) \geq \mu(a)$. Passing to a subsequence, we may assume that $\lim _{k \rightarrow \infty} \mu\left(a^{k}\right)$ exists, and there exist $\alpha^{k}:=\left\{\alpha_{j}^{k}\right\}_{1 \leq j \leq N} \subset \mathbb{D}$, for $k \geq 1$ such that $\lim _{k \rightarrow \infty} \mu\left(a^{k}\right)=\lim _{k \rightarrow \infty} \frac{1}{M_{\alpha^{k}}^{\mathbb{D}}}$. It will be enough to prove the following.

Proposition 2.9. Suppose that $\alpha^{k}=\left\{\alpha_{j}^{k}, j \in J\right\}, k \geq 0, J$ a finite set, and $\lim _{k \rightarrow \infty} M_{\alpha^{k}}^{\mathbb{D}}=M>0$.

Then there exist a sequence $\left\{\Psi_{k}\right\} \subset$ Aut $\mathbb{D}$, a subset $J^{\prime} \subset J$, and an increasing map $k \mapsto n(k)$ from $\mathbb{N}$ to $\mathbb{N}$ such that

$$
\lim _{k \rightarrow \infty} \Psi_{n(k)}\left(\alpha_{j}^{n(k)}\right)=\widetilde{\alpha}_{j} \in \mathbb{D}, \quad \forall j \in J^{\prime},
$$

and

$$
M_{\alpha^{\prime}}^{\mathbb{D}}=\lim _{k \rightarrow \infty} M_{\left\{\Psi_{n(k)}\left(\alpha_{j}^{n(k)}\right), j \in J^{\prime}\right\}}^{\mathbb{D}\}}=M .
$$


End of proof of the Theorem 2.8: Suppose

$$
\frac{1}{M}:=\liminf _{k \rightarrow \infty} \mu\left(a^{k}\right)<\mu(a) .
$$

Then $\left\{\alpha_{j}, j \in J^{\prime}\right\}$ provides a sequence mapping by $\varphi$ to $\left\{a_{j}, j \in J^{\prime}\right\}$, and $\frac{1}{M_{\left\{\alpha_{j}, j \in J\right\}}^{\mathbb{D}}}<\mu(a)$, which contradicts Theorem 2.6.

Proof of Proposition 2.9: We will proceed by downward induction on the set $J$ of indices.

Claim. Given any $\left\{\beta_{j}^{k}, j \in J\right\}_{k \geq 0}$ as in the proposition, either

Case 1: there exists $\left\{\Psi_{k}\right\}, k \mapsto n(k)$ such that $\lim _{k \rightarrow \infty} \Psi_{n(k)}\left(\beta_{j}^{n(k)}\right)=$ $\widetilde{\alpha}_{j} \in \mathbb{D}$ for all $j \in J$ and we are done, or

Case 2: there exists $J^{\prime} \varsubsetneqq J$ and $k \mapsto n(k)$ such that $\lim _{k \rightarrow \infty} M_{\left\{\beta_{j}^{n(k)}, j \in J^{\prime}\right\}}^{\mathbb{D}}=$ $M$, in which case we apply the inductive step again.

To prove the claim, consider $\ell=\limsup _{k \rightarrow \infty}\left\{\max _{i, j \in J} d_{G}\left(\beta_{i}^{k}, \beta_{j}^{k}\right)\right\}$. If $\ell<1$ then taking $\Psi_{k}=\Phi_{\beta_{j_{1}}^{k}}$ where $j_{1} \in J$ is fixed, we have that all points remain within a fixed relatively compact disk and we can choose a convergent subsequence, so we are in the first case.

Suppose now $\ell=1$. Then, passing to a subsequence, we may assume that there are $j_{1} \neq j_{2} \in J$ such that $\lim _{k \rightarrow \infty} d_{G}\left(\beta_{j_{1}}^{k}, \beta_{j_{2}}^{k}\right)=1$. Enumerate $J=\left\{j_{1}, \ldots, j_{p}\right\}$ so that for $3 \leq i \leq p, j_{i} \leq j_{i+1}$. If $\limsup _{k \rightarrow \infty} d_{G}\left(\beta_{j_{1}}^{k}, \beta_{j_{i}}^{k}\right)=1$, by taking a subsequence, we may assume that $\lim _{k \rightarrow \infty} d_{G}\left(\beta_{j_{1}}^{k}, \beta_{j_{i}}^{k}\right)=1$; we will say that $j_{i} \notin J_{1}$. If $\lim \sup d_{G}\left(\beta_{j_{1}}^{k}, \beta_{j_{i}}^{k}\right)<$ 1 , keep the same sequence and say that $j \in J_{1}$.

After a finite number of steps, we have a sequence $\left\{\beta_{j}^{n(k)}, j \in J\right\}$ and a subset $J_{1}:=\left\{j \in J: \lim \sup d_{G}\left(\beta_{j_{1}}^{k}, \beta_{j}^{k}\right)<1\right\}$ such that $j_{1} \in J_{1}$ and $j_{2} \in J_{2}:=J \backslash J_{1}=\left\{j \in J: \lim \sup d_{G}\left(\beta_{j_{1}}^{k}, \beta_{j}^{k}\right)=1\right\}$. Thus, if we set $\Psi_{k}=\Phi_{\beta_{j_{1}}^{k}}$, we have $\left\{\Psi_{n(k)}\left(\beta_{j}^{n(k)}\right), j \in J_{1}\right\}$ contained in a relatively compact disk, and $\lim _{k \rightarrow \infty}\left|\Psi_{n(k)}\left(\beta_{j}^{n(k)}\right)\right|=1, j \in J_{2}$. Passing to a further subsequence, we may assume that $\lim _{k \rightarrow \infty} \Psi_{n(k)}\left(\beta_{j}^{n(k)}\right) \in \overline{\mathbb{D}}$, for all $j \in J$. Corollary 2.4 now shows that

$$
M=\lim _{k \rightarrow \infty} \max \left(M_{\left\{\Psi_{n(k)}\left(\beta_{j}^{n(k)}\right), j \in J_{1}\right\}}^{\mathbb{D}}, M_{\left\{\Psi_{n(k)}\left(\beta_{j}^{n(k)}\right), j \in J_{2}\right\}}^{\mathbb{D}}\right),
$$


therefore, once more passing to a subsequence, there is a set $J_{i}, i=1$ or 2 , such that $\lim M_{\left\{\Psi_{n(k)}\left(\beta_{j}^{n(k)}\right), j \in J_{i}\right\}}^{\mathbb{D}}=M$. We pick $J^{\prime}=J_{i}$ for that $i$ and this proves our claim.

\section{Convergence of the function $\mu$ along finite subsequences}

Fist we would like to present the "infinite version" of Lemma 2.5.

Lemma 3.1. Suppose that $a$ is an infinite sequence in $\mathbb{B}^{n}$ and $\varphi$ is a holomorphic map from $\mathbb{D}$ to $\mathbb{B}^{n}$, and, for $1 \leq j \leq N, \alpha_{j} \in \mathbb{D}$ such that $\varphi\left(\alpha_{j}\right)=a_{j}$. Then, given any $\varepsilon>0$ there exists a holomorphic map $\psi$ from $\mathbb{D}$ to $\mathbb{B}^{n}$ and a sequence $\beta \subset \mathbb{D}$ such that

$$
\psi\left(\beta_{j}\right)=a_{j} \quad, \forall j \in Z_{+} \quad \text { and } \quad M_{\beta}^{\mathbb{D}} \geq M_{\left(\left\{\alpha_{j}\right\}_{1 \leq j \leq N}\right)}^{\mathbb{D}}-\varepsilon .
$$

Proof: Choose numbers $\varepsilon_{i}>0, i=1,2, \ldots$ such that $\sum_{1}^{\infty} \varepsilon_{i} \leq \varepsilon$. By applying Lemma 2.5 and using induction, for $1 \leq i \leq k$ we can find $\left\{\alpha_{j}^{k}\right\}_{1 \leq j \leq N+k} \subset \mathbb{D}$, and a holomorphic map $\varphi^{k}$ from $\mathbb{D}$ to $\mathbb{B}^{n}$ such that $\varphi^{k}\left(\alpha_{j}^{k}\right)=a_{j}$ for $1 \leq j \leq N+k$ and

$$
M_{\left(\left\{\alpha_{j}^{k}\right\}_{1 \leq j \leq N+k}\right)}^{\mathbb{D}} \geq M_{\left(\left\{\alpha_{j}\right\}_{1 \leq j \leq N}\right)}^{\mathbb{D}}-\sum_{i=1}^{k} \varepsilon_{i} .
$$

From the proof of Lemma 2.5, for $\varepsilon$ small enough, we have

$$
M_{\left(\left\{\alpha_{j}^{i}\right\}_{1 \leq j \leq N}\right)}^{\mathbb{D}} \geq M_{\left(\left\{\alpha_{j}^{i-1}\right\}_{1 \leq j \leq N}\right)}^{\mathbb{D}}-\varepsilon_{i}, \forall i \in Z_{+}^{*}
$$

and $\left|\alpha_{j}^{k}\right|=r_{k}^{-1}\left|\alpha_{j}^{k-1}\right|$, where we may take $r_{k}$ as close to 1 as we wish. This implies

$$
1-\left|\alpha_{j}^{k}\right|=1-r_{k}^{-1}\left|\alpha_{j}^{k-1}\right| \geq 1-\left|\alpha_{j}^{k-1}\right|-\left(1-r_{k}\right) .
$$

Choose $1-r_{k} \leq \nu_{k} \min \left\{1-\left|\alpha_{j}^{k-1}\right|, j \leq N+k-1\right\}$, where $0<\nu_{k}<1$. Then $1-\left|\alpha_{j}^{k}\right| \geq 1-\left|\alpha_{j}^{k-1}\right|-\nu_{k}\left(1-\left|\alpha_{j}^{k-1}\right|\right)=\left(1-\left|\alpha_{j}^{k-1}\right|\right)\left(1-\nu_{k}\right)$. By induction, $1-\left|\alpha_{j}^{k}\right| \geq\left(1-\left|\alpha_{j}\right|\right) \prod_{\ell=(j-N)_{+}+1}^{k}\left(1-\nu_{\ell}\right)$. Choose $\nu_{\ell}$ such that $\sum \nu_{\ell}<\infty$, then $\prod_{\ell=1}^{\infty}\left(1-\nu_{\ell}\right)=c$, a positive constant, and $1-\left|\alpha_{j}^{k}\right| \geq$ $c\left(1-\left|\alpha_{j}\right|\right)$. Thus $\left|\alpha_{j}^{k}\right| \leq 1-c\left(1-\left|\alpha_{j}\right|\right)<1, \forall k \geq 1$. By taking subsequences if necessary, we can assume that $\alpha_{j}^{k} \longrightarrow \beta_{j} \in \mathbb{D}$ as $k \rightarrow \infty$, $\forall j \geq 1$ and $\varphi^{k} \rightarrow \psi$ as $k \rightarrow \infty$ and $\psi\left(\beta_{j}\right)=a_{j}$. 
By $(*)$ we have

$$
\begin{aligned}
M_{\beta}^{\mathbb{D}} & \geq M_{\left(\left\{\alpha_{j}\right\}_{1 \leq j \leq N}\right)}^{\mathbb{D}}=\lim _{k \rightarrow \infty} M_{\left(\left\{\alpha_{j}^{k}\right\}_{1 \leq j \leq N}\right)}^{\mathbb{D}} \\
& \geq \lim _{k \rightarrow \infty}\left(M_{\left(\left\{\alpha_{j}\right\}_{1 \leq j \leq N}\right)}^{\mathbb{D}}-\varepsilon_{1}-\cdots-\varepsilon_{k}\right) \geq M_{\alpha}^{\mathbb{D}}-\varepsilon .
\end{aligned}
$$

Theorem 3.2. Let $a=\left\{a_{k}, k \in Z_{+}^{*}\right\} \subset \mathbb{B}^{n}$. Then

$$
\mu(a)=\lim _{N \rightarrow \infty} \mu\left(\left\{a_{j}\right\}_{1 \leq j \leq N}\right)=\inf _{N \in Z_{+}^{*}} \mu\left(\left\{a_{j}\right\}_{1 \leq j \leq N}\right) .
$$

Proof: Since $\mu\left(\left\{a_{j}\right\}_{1 \leq j \leq N}\right)$ is decreasing, the limit exists and equals the infimum.

For any $\varepsilon>0$, take $\alpha=\left\{\alpha_{j}\right\}_{1 \leq j \leq \infty} \subset \mathbb{D}$ such that $\frac{1}{M_{\alpha}^{\mathbb{D}}}<\mu(a)+\varepsilon$. Then there exists $N \in Z_{+}^{*}$ such that

$$
\frac{1}{M_{\left(\left\{\alpha_{j}\right\}_{1 \leq j \leq N}\right)}^{\mathbb{D}}}<\mu(a)+2 \varepsilon .
$$

Since $\mu\left(\left\{a_{j}\right\}_{1 \leq j \leq N}\right) \leq \mu(a)+2 \varepsilon$, it implies that

$$
\mu(a) \geq \inf _{N \in Z_{+}^{*}} \mu\left(\left\{a_{j}\right\}_{1 \leq j \leq N}\right) .
$$

By choosing a sequence $\alpha=\left\{\alpha_{j}\right\}_{1 \leq j \leq N}$ such that $\frac{1}{M_{\alpha}^{\mathbb{D}}}<\mu\left(\left\{a_{j}\right\}_{1 \leq j \leq N}\right)+\varepsilon$ and applying the above lemma, we have, for $\varepsilon \leq M_{\alpha}^{\mathbb{D}}-\frac{1}{2}$,

$$
\mu(a) \leq \frac{1}{M_{\beta}^{\mathbb{D}}} \leq \frac{1}{M_{\left(\left\{\alpha_{j}\right\}_{1 \leq j \leq N}\right)}^{\mathbb{D}}}+2 \varepsilon \leq \mu\left(\left\{a_{j}\right\}_{1 \leq j \leq N}\right)+3 \varepsilon,
$$

which proves the theorem.

Lemma 3.3. Let $\alpha^{\prime}$ be a subsequence of a sequence $\alpha=\left\{\alpha_{j}\right\}_{1 \leq j \leq N}$ of points in $\mathbb{D}\left(\alpha^{\prime} \varsubsetneqq \alpha\right)$. Then $M_{\alpha}^{\mathbb{D}}>M_{\alpha^{\prime}}^{\mathbb{D}}$.

Proof: Without loss of generality we may assume that $\alpha^{\prime}=\left\{\alpha_{j}\right\}_{1 \leq j \leq N-1}$. Put

$$
\begin{aligned}
R_{0}:=\frac{1}{M_{\alpha^{\prime}}^{\mathbb{D}}}= & \sup \left\{R>0: \forall w_{1}^{\prime}, \ldots, w_{N-1}^{\prime} \in \bar{D}(0, R), \exists f \in H(\mathbb{D}, \mathbb{D})\right. \\
& \left.\operatorname{such} \text { that } f\left(\alpha_{j}\right)=w_{j}^{\prime} \text { for } 1 \leq j \leq N-1\right\} \\
= & \sup \left\{R>0: \forall w_{1}^{\prime}, \ldots, w_{N-1}^{\prime} \in \bar{D}(0, R), \quad A\left(\alpha^{\prime}, w^{\prime}\right) \geq 0\right\}
\end{aligned}
$$


where $A\left(\alpha^{\prime}, w^{\prime}\right)$ denotes the quadratic form given by

$$
A\left(\alpha^{\prime}, w^{\prime}\right)(t)=\sum_{j, k=1}^{N-1} \frac{1-w_{j}^{\prime} \bar{w}_{k}^{\prime}}{1-\alpha_{j} \bar{\alpha}_{k}} t_{j} \bar{t}_{k} \quad \text { for } t \in \mathbb{C}^{N-1}(\text { see }[\mathbf{G 1}, \text { p. } 7]),
$$

and we write $Q \geq 0$ (resp. $Q>0$ ) if the quadratic form $Q$ is non negative (resp. positive).

Suppose that $A\left(\alpha^{\prime}, w^{\prime}\right)>0, \forall w^{\prime}=\left(w_{1}^{\prime}, \ldots, w_{N-1}^{\prime}\right) \in \bar{D}\left(0, R_{0}\right)^{N-1}$. Consider the open subset $\Omega:=\left\{w^{\prime}: A\left(\alpha^{\prime}, w^{\prime}\right)>0\right\}$ of $\mathbb{D}^{N-1}$. Since $\Omega \supset \bar{D}\left(0, R_{0}\right)^{N-1}$, it implies that there exists $R^{\prime}>R_{0}$ such that $\Omega \supset$ $\bar{D}\left(0, R^{\prime}\right)^{N-1}$. It is impossible.

So there exists $w^{\prime} \in \bar{D}\left(0, R_{0}\right)^{N-1}$ such that $A\left(\alpha^{\prime}, w^{\prime}\right)$ is degenerate. Consider

$$
\begin{aligned}
& W:=\left\{w_{N}: \exists f \in H(\mathbb{D}, \mathbb{D}) \text { such that } f\left(\alpha_{N}\right)=w_{N}\right. \\
& \left.\qquad \text { and } f\left(\alpha_{j}\right)=w_{j}^{\prime}, 1 \leq j \leq N-1\right\} .
\end{aligned}
$$

If $M_{\alpha}^{\mathbb{D}}=M_{\alpha^{\prime}}^{\mathbb{D}}=\frac{1}{R_{0}}$ then $W \supset \bar{D}\left(0, R_{0}\right)$. But $A\left(\alpha^{\prime}, w^{\prime}\right)$ is degenerate, thus $W$ is a single point (see [G1]). It is a contradiction. Thus $M_{\alpha}^{\mathbb{D}}>$ $M_{\alpha^{\prime}}^{\mathbb{D}}$.

Lemma 3.4. Let $a=\left\{a_{j}\right\}_{1 \leq j \leq N} \subset \mathbb{B}^{n}$. If $\mu(a)<\min \left\{\mu\left(a^{\prime}\right): a^{\prime} \varsubsetneqq a\right\}$ then there exist a sequence $\alpha=\left\{\alpha_{j}\right\}_{1 \leq j \leq N} \subset \mathbb{D}$ and a holomorphic map $\varphi$ from $\mathbb{D}$ to $\mathbb{B}^{n}$ such that $\varphi\left(\alpha_{j}\right)=a_{j}$ for $1 \leq j \leq N$ and $\mu(a)=$ $\frac{1}{M_{\alpha}^{\mathbb{D}}}$.

Proof: Let $\varphi^{k}, \alpha^{k}=\left\{\alpha_{j}^{k}\right\}_{1 \leq j \leq N} \subset \mathbb{D}$ such that $\varphi^{k}\left(\alpha_{j}^{k}\right)=a_{j}, 1 \leq j \leq N$ and $\frac{1}{M_{\alpha^{k}}^{\mathbb{D}}}$ tends to $\mu(a)$.

Taking a subsequence, we may now assume that $\alpha^{k}$ converges pointwise to a sequence $\alpha \in \overline{\mathbb{D}}$. By Montel's theorem, without loss of generality we may assume that the subsequence $\varphi^{k}$ converges to $\varphi$ uniformly on compact sets. It is easy to see that $\varphi\left(\alpha_{j}\right)=a_{j}$ for any $1 \leq j \leq N$ with $\left|\alpha_{j}\right|<1$ and $\varphi(\mathbb{D}) \subset \overline{\mathbb{B}}^{n}$. In fact, $\varphi(\mathbb{D}) \subset \mathbb{B}^{n}$, by the maximum principle for a strictly convex ball. 
If $\left|\alpha_{j}\right|<1$ for all $1 \leq j \leq N$, then the proof of lemma is completed. Now suppose that there exists $j$ such that $\left|\alpha_{j}\right|=1$. By applying Proposition 2.9 we have a subset $J^{\prime} \varsubsetneqq\{1, \ldots, N\}$ such that

$$
\frac{1}{M_{\left(\left\{\alpha_{j}^{k}\right\}_{1 \leq j \leq N}\right)}^{\mathbb{D}}} \text { and } \frac{1}{M_{\left(\left\{\alpha_{j}^{k}\right\}_{j \in J^{\prime}}\right)}^{\mathbb{D}}}
$$

tend to $\mu(a)$, so that $\mu(a)=\mu\left(a^{\prime}\right)$ with $a^{\prime}=\left\{a_{j}\right\}_{j \in J^{\prime}}$. It is impossible.

Theorem 3.5. For any $N \in Z_{+}^{*}$ there exists an open set $U$ of sequences a with $\# a=N$ such that for every $a \in U$ there is $\alpha=\left\{\alpha_{j}\right\}_{1 \leq j \leq N} \subset \mathbb{D}$ such that $\varphi\left(\alpha_{j}\right)=a_{j}$ for $1 \leq j \leq N$ and $\mu(a)=\frac{1}{M_{\alpha}^{\mathbb{D}}}$.

Proof: Now we give a sequence satisfying the sufficient condition of Lemma 3.4.

Let $a=\left\{a_{j}\right\}_{1 \leq j \leq N} \subset \mathbb{B}^{n}$ lie on a complex line $\Delta$. We may assume that $\Delta=\mathbb{C}_{\times}\{0, \ldots, 0\}$ and $a_{j}=\left(a_{j}, 0, \ldots, 0\right)$ for $1 \leq j \leq N$. Consider a holomorphic map $\varphi$ from $\mathbb{D}$ to $\mathbb{B}^{n}$ given by $\varphi(\zeta)=(\zeta, 0,0, \ldots, 0)$. Take $\alpha=\left\{a_{j}\right\}_{1 \leq j \leq N} \subset \mathbb{D}$. It implies that $\varphi(\alpha)=a$ and $M_{\alpha}^{\mathbb{D}}=M_{a}^{\mathbb{B}^{n}}$. Thus $\mu(a)=\frac{1}{M_{a}^{\mathbb{B}^{n}}}=\frac{1}{M_{\alpha}^{\mathbb{D}}}$. By applying Lemma 3.3 we have $M_{a^{\prime}}^{\mathbb{B}^{n}}<M_{a}^{\mathbb{B}^{n}}$ for $a^{\prime} \varsubsetneqq a$. Then $\mu\left(a^{\prime}\right)>\mu(a)$ and, hence $\mu(a)<\min \left\{\mu\left(a^{\prime}\right): a^{\prime} \varsubsetneqq a\right\}$.

Now let $b$ be another sequence close to $a$. Since $\min \left\{\mu\left(b^{\prime}\right): b^{\prime} \varsubsetneqq b\right\}$ depends continuously on $b$ and $\mu(b)$ is continuous, the map $b \longmapsto \mu(b)-$ $\min \left\{\mu\left(b^{\prime}\right), b^{\prime} \varsubsetneqq b\right\}$ is continuous. Since the value of this function at $b=a$ is negative, there exists a neighborhood $U$ of $a$ in $\left(\mathbb{B}^{n}\right)^{N}$ such that $\mu(b)<\min \left\{\mu\left(b^{\prime}\right): b^{\prime} \varsubsetneqq b\right\}$ for all $b \in U$. The proof of Theorem 3.5 will be completed by applying Lemma 3.4 to $b$.

Theorem 3.6. Let $a=\left\{a_{j}\right\}_{1 \leq j \leq N}$ be a sequence of points in $\mathbb{B}^{n}$. If there exists a holomorphic map $\varphi_{0}$ from $\mathbb{D}$ to $\mathbb{B}^{n}$ and $\alpha=\left\{\alpha_{j}\right\}_{1 \leq j \leq n} \subset$ $\mathbb{D}$ such that $\varphi_{0}\left(\alpha_{j}\right)=a_{j}$ for $1 \leq j \leq N$ and $\mu(a)=\frac{1}{M_{\alpha}^{\mathbb{D}}}$, then $\varphi_{0}$ is a solution of the extremal problem

$$
\inf \left\{\|\psi\|_{\infty}: \psi \in H\left(\mathbb{D}, \mathbb{C}^{n}\right), \psi\left(\alpha_{j}\right)=a_{j} \text { for } 1 \leq j \leq N\right\} .
$$


Proof: If $\varphi_{0}$ is not of minimal norm then there is another holomorphic map $\eta$ from $\mathbb{D}$ to $\mathbb{B}^{n}$ and $r<1$ such that $\eta\left(r \alpha_{j}\right)=a_{j}$ for $1 \leq j \leq N$ (see $\left[\mathbf{A}-\mathbf{T}\right.$, Lemma 7]). It is easy to see that $M_{r \alpha}^{\mathbb{D}} \geq M_{\alpha}^{\mathbb{D}}$. Now we prove $M_{r \alpha}^{\mathbb{D}}>M_{\alpha}^{\mathbb{D}}$.

For any $\varepsilon>0$, by the definition of $M_{\alpha}^{\mathbb{D}}$ there exist $v_{1}, \ldots, v_{N} \in \mathbb{D}$ such that $I:=\inf \left\{\|f\|_{\infty}: f\left(\alpha_{j}\right)=v_{j}, 1 \leq j \leq N\right\} \geq M_{\alpha}^{\mathbb{D}}-\varepsilon$ and there exists a map $f_{\varepsilon} \in H^{\infty}(\mathbb{D})$ such that $\left\|f_{\varepsilon}\right\|_{\infty} \leq M_{r \alpha}^{\mathbb{D}}+\varepsilon$ and $f_{\varepsilon}\left(r \alpha_{j}\right)=v_{j}$ for $1 \leq j \leq N$.

Put $g_{\varepsilon}(\zeta)=f_{\varepsilon}(r \zeta)$. We have $g_{\varepsilon}\left(\alpha_{j}\right)=v_{j}$ and $\left\|g_{\varepsilon}\right\|_{\infty} \leq \sup \left|f_{\varepsilon}\right|$. Now we estimate this last quantity:

$$
\frac{1}{M_{r \alpha}^{\mathbb{D}}+\varepsilon} f_{\varepsilon}\left(r \alpha_{1}\right)=\frac{v_{1}}{M_{r \alpha}^{\mathbb{D}}+\varepsilon} \in \bar{D}\left(0, \frac{1}{M_{r \alpha}^{\mathbb{D}}+\varepsilon}\right) \subset \bar{D}\left(0, \frac{1}{M_{r \alpha}^{\mathbb{D}}}\right) \subset \mathbb{D} .
$$

By Schwarz's lemma, $f_{\varepsilon}\left(r \alpha_{1}\right)=v_{1}$ implies that

$$
d_{G}\left(\frac{f_{\varepsilon}(\zeta)}{M_{r \alpha}^{\mathbb{D}}+\varepsilon}, \frac{v_{1}}{M_{r \alpha}^{\mathbb{D}}+\varepsilon}\right) \leq d_{G}\left(\zeta, r \alpha_{1}\right) \leq C<1
$$

for any $\zeta \in \bar{D}(0, r)$. Thus, for $\zeta \in \bar{D}(0, r)$,

$$
\frac{f_{\varepsilon}(\zeta)}{M_{r \alpha}^{\mathbb{D}}+\varepsilon} \in B_{G}\left(\frac{v_{1}}{M_{r \alpha}^{\mathbb{D}}+\varepsilon}, C\right):=\left\{\xi: d_{G}\left(\frac{v_{1}}{M_{r \alpha}^{\mathbb{D}}+\varepsilon}, \xi\right) \leq C\right\} .
$$

On the other hand, we see that

$$
B_{G}\left(\frac{v_{1}}{M_{r \alpha}^{\mathbb{D}}+\varepsilon}, C\right) \subset \bigcup_{|\xi|<\frac{\left|v_{1}\right|}{M_{r \alpha}^{\mathbb{D}}}} B_{G}(\xi, C) \subset \mathbb{D}\left(0, \frac{C+\frac{\left|v_{1}\right|}{M_{r \alpha}^{\mathbb{D}}}}{1+C \frac{\left|v_{1}\right|}{M_{r \alpha}^{\mathbb{D}}}}\right) .
$$

Put

$$
C^{\prime}:=\frac{C+\frac{\left|v_{1}\right|}{M_{r \alpha}^{\mathbb{D}}}}{1+C \frac{\left|v_{1}\right|}{M_{r \alpha}^{\mathbb{D}}}}<1 .
$$

Then $M_{\alpha}^{\mathbb{D}}-\varepsilon \leq I \leq\left\|g_{\varepsilon}\right\|_{\infty} \leq C^{\prime}\left(M_{r \alpha}^{\mathbb{D}}+\varepsilon\right)$. It implies that $C^{\prime}\left(M_{r \alpha}^{\mathbb{D}}+\right.$ $\varepsilon) \geq M_{\alpha}^{\mathbb{D}}-\varepsilon$. Since $C^{\prime}<1, C^{\prime} M_{r \alpha}^{\mathbb{D}}>M_{\alpha}^{\mathbb{D}}-2 \varepsilon$ for any $\varepsilon>0$.

Thus $C^{\prime} M_{r \alpha}^{\mathbb{D}} \geq M_{\alpha}^{\mathbb{D}}$ and hence $M_{r \alpha}^{\mathbb{D}}>M_{\alpha}^{\mathbb{D}}$.

Acknowledgement. The results of this paper were done under the guidance of Prof. Pascal J. Thomas. We would like to thank him and Prof. Do Duc Thai for the stimulating discussions we had regarding this paper. 


\section{References}

[A-T] E. Amar and P. J. Thomas, A notion of extremal analytic discs related to interpolation in the ball, Math. Ann. 300 (1994), 419-433.

[B] B. Berndtsson, Interpolating sequences for $H^{\infty}$ in the ball, Nederl. Akad. Wetensch. Indag. Math. 88 (1985), 1-10.

[B-C-L] B. Berndtsson, S. A. Chang and K. C. Lin, Interpolating sequences in the polydisc, Trans. Amer. Math. Soc. 302 (1987), $161-169$.

[C] L. Carleson, An interpolation problem for bounded analytic functions, Amer. J. Math. 80 (1958), 921-930.

[G1] J. B. Garnett, "Bounded Analytic Functions", Academic Press, Inc. New York-London, 1981.

[G2] J. GLOBEvnik, Discs in the ball containing given discrete sets, Math. Ann. 281 (1988), 87-96.

[R] W. Rudin, "Function Theory in the Unit Ball of $\mathbb{C}^{n}$ ", Springer Verlag, New York-Berlin, 1980.

[T1] P. J. Thomas, Continuity and convergence properties of extremal- interpolating disks, Publ. Mat. 39 (1995), 335-347.

[T2] P. J. Thomas, Necessary conditions for interpolating sequences, Bull. Soc. Math. (to appear).

[V] N. Th. Varopoulos, Sur un problème d'interpolation, $C . R$. Acad. Sci. Paris 274 (1972), 1539-1542.

Department of Mathematics

Pedagogical University of Hanoi

Hanoi

Vietnam

E-mail address: trang@netnam.org.vn

Primera versió rebuda el 18 de desembre de 1998, darrera versió rebuda el 3 de juny de 1999. 